\title{
Call Admission Control for Next Generation Wireless Networks Using Higher Order Markov Model
}

\author{
Ramesh Babu H.S \\ Department of Information Science and Engineering, Acharya Institute of Technology \\ E-mail: rameshbabu@acharya.ac.in \\ Gowrishankar \\ Department of Computer Science and Engineering, B.M.S. College of Engineering \\ E-mail: gowrishankar.cse@bmsce.ac.in
}

Satyanarayana P.S

Department of Electronics and Communication Engineering, B.M.S. College of Engineering,

Bangalore, INDIA

E-mail: pssvittala.ece@bmsce.ac.in

\begin{abstract}
The Next generation wireless networks (NGWN) will be heterogeneous which will have different radio access technologies (RATs) operating together. The Radio Resource Management (RRM) is one of the key challenges in NGWN. The Call admission control (CAC) mechanism is one of the Radio Resource Management technique plays instrumental role in ensuring the desired QoS to the users working on different applications which are having the diversified nature of QoS requirements to be fulfilled by the wireless networks. One of the key challenges to be addressed in this prevailing scenario is the distribution of the available channel capacity amongst the multiple traffic with different bandwidth requirements so as to guarantee the QoS requirements of the traffic .The call blocking probability is one such QoS parameter for the wireless network and for better QoS it is desirable to reduce the call blocking probability. In this customary scenario it is highly advantageous to bring about an analytic Performance model. In this paper we propose a call admission control framework based on higher order Markov chains to effectively handle the call blocking probability in NGWN and to provide optimal QoS for the mobile users. In the proposed algorithm we have considered three classes of traffic having different QoS requirements. The results obtained from the Performance model are encouraging and optimistic and indicates the need of an intelligent decision making system for CAC.
\end{abstract}

Keywords: Radio Resource Management, Call admission control, Call blocking probability, QoS

\section{Introduction}

The recent advances in the wireless networks and mobile devices are inclined towards emerging of ubiquitous computing where the users and applications running in the mobile terminal (MT) can enjoy seamless roaming. It is well known that the basic problem in the wireless networks is the scarce of the radio resources. The efficient radio resource management is very essential. The admission control is one of the radio resource management technique which plays dominant role in effectively managing the resources. The admission control in the wireless networks will reduce the call blocking probability in the wireless networks by optimizing the utilization of the available radio resources. The mobile communication environment is featured by moving terminals with different QoS requirements and in this current scenario the need of guaranteed QoS is more sought for.

The future users of mobile communications look for always best connected (ABC) networks at anywhere and anytime among the complementary access technologies like wireless local area networks (WLAN), and worldwide inter operability for microwave access (Wi-MAX), Global Systems for Mobile Communications(GSM), General Packet Radio Service(GPRS), Universal mobile telecommunication systems(UMTS) etc.The mobile communication networks are evolving into adaptable Internet protocol based networks that can handle multimedia applications. When the multimedia data is supported by wireless networks, the networks should meet the quality of service requirements. One of the key challenges to be addressed in this prevailing scenario is the distribution of the available channel capacity among the multiple traffic that are with different bandwidth requirements. 
The existing admission control strategies can handle the resource management in homogeneous wireless networks but are unable to effectively handle the issues in heterogeneous wireless environment. The mobility of the terminals in the mobile communication environment makes the resource allocation a challenging task when the resources are always in scarce. The efficient call admission control policies should be in place which can take care of this contradicting environment to optimize the resource utilization.

The design of call admission control algorithm must take into consideration packet level QoS parameters like minimum delay, jitter as well as session level QoS parameters like call blocking probability (CBP) and call dropping probability (CDP). The CBP is the probability of denial of accepting the new call and CDP the likelihood of dropping the call by a new access network due to decline of the network resources to an unacceptable level in other words the networks is exhausted with the available resources at which it drops the handover calls. In mobile networks the admission control traffic management mechanism is needed to keep the call blocking probability at a minimal level and another RRM strategy vertical handovers plays crucial role in reducing the and call dropping probability in an heterogeneous wireless networks.

In further sections of the paper is organized as follows. The section II discusses on the motivation and related work. Section III focuses on the proposed system model for the call admission control based on higher order Markov chains. The section IV represents the simulation results and conclusion and future work is indicated in section V.

\section{Related Work}

At present, dissimilar wireless access networks including 2.5G, 3G, Bluetooth, WLAN and Wi-Max coexist in the mobile computing environment, where each of these Radio access technologies offer complementary characteristics and features in terms of its coverage area, data rate, resource utilization, power consumption etc.. With all these there are constant improvements in the existing technologies offering better performance at lesser cost. This is beneficial in both the end users and service provider's perspective. The idea of benefiting from integrating the different technologies has lead to the concept of beyond International mobile telephony 2000(IMT-2000) wireless networks known as the next generation wireless networks $(\mathrm{NGWN})$. In this heterogeneous environment, the end user is expected to be able to connect to any of the different available access networks. The end user will also be able to roam seamlessly within these access networks through vertical handover mechanisms. The global roaming is supplemented by the existence of IP networks as the backbone which makes the mobile computing environment to grow leaps and bounds and can effectively address the issue with regard to converge limitations is concerned. In this multifaceted wireless radio environment the radio resource management plays major role. The effective utilization of the limited available resources is the challenge. The admission control is one such challenge a network service provider face to achieve better system utilization face in handling this complex scenario to provide the best QoS to the users of the network.

Call admission control schemes can be divided into two Categories, local and collaborative schemes [1]. Local schemes use local information alone (e.g. local cell load) when taking the admission decision. Examples of these schemes are(T. Zhang, E.v.d. Berg, J. Chennikara, P. Agrawal, J.-C. Chen, T. Kodama, 2001)(C.-T. Chou, K.G. Shin, 2002)(C.W. Ahn, R.S. Ramakrishna, 2004). Collaborative schemes involve more than one cell in the admission process. The cells exchange information about the ongoing sessions and about their capabilities to support these sessions (T. Zhang, E.v.d. Berg, J. Chennikara, P. Agrawal, J.-C. Chen, T. Kodama, 2001). The fundamental idea behind all collaborative admission control schemes is to consider not only local information but also information from other cells in the network. The local cell, where the new call has been requested, communicates with a set of cells that will participate in the admission process. This set of cells is usually referred to as a cluster. In general, the schemes differ from each other according to how the cluster is constructed, the type of information exchanged and how this information is used. In (M. Naghshineh, M. Schwartz, 1996) for example, the cluster is defined as the set of direct neighbors. The main idea is to make the decision of admission control in a decentralized manner.

There are good amount of work reported for homogenous wireless networks and single service wireless networks and few works in the heterogeneous wireless networks. The Call admission control in Heterogeneous wireless networks is a real challenge. The varied QoS requirements of multimedia applications and the coexistence of different RATs, facade major challenges in designing CAC algorithms for next generation heterogeneous wireless networks. The challenges are heterogeneous networking, multiple service classes, flexibility in bandwidth allocation and cross layer issues based design(Ramesh Babu H.S., Gowrishankar, Satyanarayana P.S. 2009).

\subsection{Heterogeneous networking}

4G networks will have different types of RATs different from each other by air interface technology, cell size, services, price, access method, coverage, so CAC schemes must be able to handle new type of handoff called vertical handoff.

\subsection{Multiple service classes}

The B3G networks should be able to accommodate the applications and user with different QoS requirements, so the CAC algorithms should be designed to handle different classes of service to meet the QoS needs of all types of 
applications.

\subsection{Flexible in bandwidth allocation}

The diversity is in multimedia applications and mobile users QoS requirements in NGWN. The resource utilization and QoS performance can be improved by adaptive bandwidth allocation. This clearly indicates that the CAC should be designed taking into consideration the flexible bandwidth allocation, where, more resources can be allocated when the there is less traffic and the allocated bandwidth can be revoked when there is congestion.

\subsection{Cross layer issues based design}

The traditional CAC schemes were based on the call level QoS only and few of them have considered the physical layer QoS like SIR as QoS criteria. Unlike the tradition voice oriented circuit switched network, the next generation network predicted to be pure packet based network and the QoS needs to be addressed both at call level as well as at packet level. This mandates that the new call has to be admitted only if both call level QoS metrics like call blocking and dropping probabilities and the packet level QoS measures like packet transmission delay and packet dropping probability are maintained at some desired levels.

The other important solution for the decision making of call admission control is by multi criteria decision making (MCDM). This is an optimization technique used to analyze the contradicting decision making parameters. The MCDM based decision making systems are generally used in the fields of reliability, financial analysis, social and political related analysis and environmental impact analysis etc. The NGWN has different RATs coexisting which are with different capabilities and they should cater the varied QoS requirements of multimedia applications admission control with single criteria may be too trivial, in this prevailing scenario the admission control decision should be based on Multi criteria such that the optimization user satisfaction and selection of optimal RAT is achieved. There are several algorithms proposed on handling the admission control decision making using MCDM in heterogeneous wireless networks.

There are Different admission control algorithms based on multiple criteria decision making. They are categorized as Utility-function based CAC and computation Intelligence CAC. In the Utility-function based CAC the incoming calls are admitted based on some utility or cost function based on multiple criteria. These algorithms are very optimal algorithms and in most of the case are complex in nature and pose high computational overhead.

The computation-Intelligence-based CAC use evolutionary approaches like Genetic Algorithm (GA), fuzzy logic and Artificial Neural Networks(ANN) (R.T.Marler and J.S. Arora, 2004).Majority of the computational-intelligence-based CAC algorithms incorporate fuzzy logic(P.M.L. Chan, R.E. Sheriff, Y.F. Hu, P. Conforto, C. Tocci, 2001), fuzzy neural(R. Agusti, O. Sallent, J. Pérez-Romero, L. Giupponi, 2004) and fuzzy MCDM(W. Zhang, 2004)(A.L. Wilson, A. Lenaghan, R. Malyan, 2005) methods. There are very few works reported on the usage of Artificial Neural Networks in CAC.

This paper is pitched upon the call admission control mechanism in heterogeneous networks scenario handling different types of calls in the system.

\section{System Model}

In this paper we propose a novel analytical model for admission control for the call blocking probability there by increasing the resource utilization. This would achieve the Objective of guaranteeing the user QoS requirements. The proposed model is able to handle three types of applications which are complementary in nature with respect to their QoS requirements are considered. The applications considered for the study involves conversation traffic, interactive traffic and background traffic. The representative applications could be voice calls, Web browsing and file transfer applications respectively. We have considered a heterogeneous network which comprises a set of RATs $R n$ with co-located cells in which radio resources are jointly managed. Cellular networks such as Wireless LAN and Wi-Max can have the same and fully overlapped coverage, which is technically feasible, and may also save installation cost. $\mathrm{H}$ is the set of heterogeneous wireless networks coexisting is given as $\mathrm{H}=$ \{RAT 1, RAT 2, RAT k\} and where K is the total number of RATs in the heterogeneous wireless network. The heterogeneous wireless network supports n-classes of calls, and each RAT in set $\mathrm{H}$ is optimized to support certain classes of calls.

The Analytical model for Call admission control mechanism in heterogeneous wireless networks is modeled using higher order Markov chain as shown in figure2.The study considers that, whenever a new user enters the network will originate the network request at the rate $\lambda_{i}$ and is assumed to follow a Poisson process. The service time of the different class of traffic and types of calls is $\mu_{i}$. The mean service time of all types of users were assumed to follow negative exponential distribution with the mean rate $1 / \mu$. Since Voice traffic is Erlang distributed and the condition that is considered for simulation is Negative Exponential distribution. The total number of virtual channels in the system are $\mathrm{N}$. When the numbers of available channels are below the specified threshold the system will block/drop the calls. The threshold limit is determined by three positive integers $A_{1}, A_{2}$ and $A_{3}$. When the number of available channels falls 
below the threshold $\boldsymbol{A}_{3}$ the proposed system will accept only the voice calls and web browsing. When the number of available channels falls below the threshold $A_{2}$ the proposed system will accept only the voice calls and when the available number of channels falls below the threshold $A_{I}$ the proposed system will not accept any calls as it reaches the stage where there will be no channels available to allocate to the incoming calls and leads to system blocking. The $P(0)$ is probability that there are no allocated channels in the designated system.

For the system model shown in figure1 the system state can be defined for the lower and upper boundaries as in (1)-(3) and (4)-(6) respectively.

The equations (1) - (3) are lower boundary equations represents the system states $P_{0}, P_{1}, P_{2}$

$$
\begin{aligned}
& \lambda_{1} P_{0}+\lambda_{2} P_{0}+\lambda_{3} P_{0}-\mu_{2} P_{2}-\mu_{1} P_{1}-\mu_{3} P_{3}=0 \\
& \lambda_{1} P_{1}+\lambda_{2} P_{1}+\lambda_{3} P_{1}-\mu_{1} P_{2}-\mu_{2} P_{3}-\mu_{3} P_{3}=0 \\
& \lambda_{1} P_{2}+\lambda_{2} P_{2}+\lambda_{3} P_{2}+\mu_{1} P_{2}+\mu_{2} P_{2}-\mu_{1} P_{3}-\mu_{2} P_{4}-\mu_{3} P_{5}=0
\end{aligned}
$$

The equations (4) - (6) are upper boundary equations for the system states $P_{n}, P_{n-1}, P_{n-2}$ and are expressed as

$$
\begin{aligned}
& P_{n-3}\left(\lambda_{1}+\lambda_{2}+\lambda_{3}+\mu_{1}+\mu_{2}+\mu_{3}\right)-\lambda_{1} P_{n-4}-\lambda_{2} P_{n-5}-\lambda_{3} P_{n-6}-\mu_{1} P_{n-2}-\mu_{2} P_{n-1}-\mu_{3} P_{n}=0 \\
& P_{n-2}\left(\lambda_{1}+\lambda_{2}+\mu_{1}+\mu_{2}+\mu_{3}\right)-\lambda_{1} P_{n-3}-\lambda_{2} P_{n-4}-\lambda_{3} P_{n-5}-\mu_{1} P_{n-1}-\mu_{2} P_{n}=0 \\
& P_{n-1}\left(\lambda_{1}+\mu_{1}+\mu_{2}+\mu_{3}\right)-\lambda_{1} P_{n-2}-\lambda_{2} P_{n-3}-\lambda_{3} P_{n-4}-\mu_{1} P_{n}=0
\end{aligned}
$$

The repeated states are those which are in-between these upper boundary and lower boundary states based on figure1. The repeated states of the system are represented in a generic form as.

$$
P_{4}\left(\lambda_{1}+\lambda_{2}+\lambda_{3}+\mu_{1}+\mu_{2}+\mu_{3}\right)-\lambda_{1} P_{3}-\lambda_{2} P_{2}-\lambda_{3} P_{5}-\mu_{1} P_{6}-\mu_{2} P_{6}-\mu_{3} P_{7}=0
$$

The equation that can be presumed as the general equation for call blocking probability for traffic type 1 is

$$
P_{n}=\frac{\lambda_{1} P_{\mathrm{n}-1}+\lambda_{2} P_{n-2}+\lambda_{3} P_{\mathrm{n}-3}}{\left(\mu_{1}+\mu_{2}+\mu_{3}\right)}
$$

Assuming that the arrival time of all the types of traffic are equal i.e. $\quad \lambda_{1}=\lambda_{2}=\lambda_{3}=\lambda$ and the service time for the types of traffic are equal i.e. $\mu_{1}=\mu_{2}=\mu_{3}=\mu$, the call blocking probability for type 1 traffic could be expressed as

$$
P_{n}=\frac{a}{3}\left(P_{\mathrm{n}-1}+P_{n-2}+P_{\mathrm{n}-3}\right)
$$

Where $\mathrm{a}=\lambda / \mu$ is called as Utilization rate which should be generally less than one for the system stability.

Similarly, the call blocking probability for type 2 traffic $P_{n-1}$ is

$$
P_{n-1}=\frac{a}{3}\left(P_{\mathrm{n}-2}+P_{n-3}+P_{\mathrm{n}-4}\right)
$$

And the call blocking probability for type 3 traffic $P_{n-2}$ is represented as

$$
P_{n-2}=\frac{a}{3}\left(P_{\mathrm{n}-3}+P_{n-4}+P_{\mathrm{n}-5}\right)
$$

The call blocking probability for the overall system traffic $P_{n b}$ can be expressed as 


$$
P_{n b}=\frac{a}{3}\left(P_{n}+P_{n-1}+P_{n-2}\right)
$$

\section{Simulation Results and Discussion}

In this section, we present the numerical results and compare the call blocking probabilities of the different types of traffic and MATLAB is used for the simulation. The proposed performance model for call admission control mechanism is analyzed for the call blocking probability by making the variation in the number of channels. .The experiment setup is conducted by varying the traffic intensity of Type1 traffic and the blocking probability of type1, blocking probability of the type2, and type 3 traffic of the system is plotted. The Figure 2 shows call blocking probability for all three types of traffic. The horizontal axis shows the number of users with type 1 traffic while the vertical axis shows the call blocking probability of all types of traffic.

The parameters of analytic performance model are also called as Performance model parameters. The Performance model parameters are number of virtual channels $(\mathrm{N})$, user arrival rate $(\lambda)$, arrival rate of type 1 call $(\lambda 1)$, arrival rate of type 2 call $\left(\lambda 2\right.$.) arrival rate of type 3 call $(\lambda 3)$ and the service time of the user $(\mu)$, service time of the call of type $1\left(\mu_{1}\right)$, Service time of the call of type $2\left(\mu_{2}\right)$, Service time of the call of type $3\left(\mu_{3}\right)$. The simulation results shows that the call blocking probability of the different types of traffic will increase with the increase in the intensity of type 1 traffic. The simulation results with increase the intensity of type 2 traffic and simulation results with increase the intensity of type 3 also showed the similar kind of results. The simulation results indicate that at particular state the call blocking probability of all three types of traffic will be reduced.

The important observations from the performance model are: Increase in the traffic of Type1 call and reduce in the type 2 and type 3 call will reduce the Call blocking probability of type 1 call. The results indicate reduction in call blocking probability of type 2 calls When reduce the type 1 and type 2 traffic in the system. Similarly when we reduce the type 1 and type 2 traffic, the call blocking probability of type 3 traffic is minimal. In a next generation wireless system, it is desirable to uphold guaranteed QoS to the all types of users and optimum Utilization of system resource needed. In order to provide superior QoS to the user/application it is required to maintain the call blocking probability minimal. The bandwidth reservation for each type of traffic may reduce the call blocking probability. But this is possible when we have non varying traffic for all the types of traffic and there is always a possibility of wastage of the bandwidth if one of the three traffic intensity is lower than the bandwidth of the allocated channels. It is predicted that the next generation network is heterogeneous and obviously we have the time varying traffic as iterated in the in the traffic model in section 4 .

In this prevailing condition it is very difficult and tricky situation to satisfy the needs of different types of traffic. Hence it is enviable to use a multi constraint optimization technique in achieving better tradeoff between set of system performance metrics. The performance parameters such as BER, ABW and network traffic are the input to the optimization technique for achieving better tradeoff between set of performance metrics. Some of the well known techniques in solving multi constraint optimization problem are Game theory, Markov Decision Process(MDP), Genetic Algorithm(GA), Goal programming and Multi attribute Decision Making (MADM) technique. Among these set of techniques MADM is a most widely used method (K.P.Yoon and C.Hwang, 1995).

\section{Conclusion and Future Work}

In this paper, we have proposed a performance model for call admission control mechanism in the heterogeneous RATs and analyzing the call blocking probability keeping the variation in the number of channels. In order to measure the call blocking probability of the analytical model the simulation study was made and following observations were made. In the experiment setup all the types of traffic was varied and Firstly, increase in the number of type 1 users will increase the call blocking probability of type 2 and type 3 calls and vice versa. Second, Increase in the traffic intensity of one type of traffic will increase the system blocking probability.

The concept of minimizing the call blocking probability is an optimization technique to provide fair QoS to the set of users in the wireless network and there is also a need of intelligent call admission control strategy in the admission control mechanism to make the decision of accepting are rejecting a call keeping the blocking probability minimal in a heterogeneous RATs based network working under dynamic network condition. The future work of this research is pitched upon using Intelligence to the call admission control decision process .The future work includes application of Fuzzy neural technique for the decision making .It is evident from the above discussion that that the application of intelligent techniques with multiple criteria for decision making is worthwhile.

\section{References}

A.L. Wilson, A. Lenaghan, R. Malyan. (2005). Optimizing wireless network selection to maintain QoS in heterogeneous wireless environments, in: Proceedings of World Communication Forum, Denmark, September, 2005.

C.-T. Chou, K.G. Shin. (2002) Analysis of combined adaptive bandwidth allocation and admission control in wireless 
networks, in: IEEE Conference on Computer Communications (INFOCOM), 2002 pp. 676-684.

C.W. Ahn, R.S. Ramakrishna. (2004). QoS provisioning dynamic connection admission control for multimedia wireless networks using a Hopfield neural network, IEEE Transactions on Vehicular Technology 53 (1)(2004) 106-117.

K.P.Yoon and C.Hwang, (1995). "Multiple attribute decision making", Sage,1995.

M. Naghshineh, M. Schwartz. (1996). Distributed call admission control in mobile/wireless networks, IEEE Journal on Selected Areas in Communications (JSAC) 14 (4) (1996) 711-717.

P.M.L. Chan, R.E. Sheriff, Y.F. Hu, P. Conforto, C. Tocci. (2001). Mobility management incorporating fuzzy logic for a heterogeneous IP environment, IEEE Communications Magazine 39 (12) (2001) 42-51.

R. Agusti, O. Sallent, J. Pe'rez-Romero, L. Giupponi. (2004). A fuzzy-neural based approach for joint radio resource management in a beyond $3 \mathrm{G}$ framework, in: 1st International Conference on Quality of Service in Heterogeneous Wired/Wireless Networks (Qshine’04), Dallas, USA, October, 2004.

R.T.Marler and J.S. Arora. (2004). "Survey of multi-objective optimization methods for engineering", Structural and multidisciplinary optimization, Vol.26.No.6,pp 369-395,2004.

Ramesh Babu H.S., Gowrishankar, Satyanarayana P.S. (2009). Call admission control approaches in beyond 3G networks using multi criteria decision making , 2009 First International Conference on Computational Intelligence, Communication Systems(CICSYN2009).

T. Zhang, E.v.d. Berg, J. Chennikara, P. Agrawal, J.-C. Chen, T. Kodama. (2001). Local predictive resource reservation for handoff in multimedia wireless IP networks, IEEE Journal on Selected Areas in Communications (JSAC) 19 (10) (2001) 1931-1941.

T. Zhang, E.v.d. Berg, J. Chennikara, P. Agrawal, J.-C. Chen, T. Kodama. (2001). Local predictive resource reservation for handoff in multimedia wireless IP networks, IEEE Journal on Selected Areas in Communications (JSAC) 19 (10) (2001) 1931-1941.

W. Zhang. (2004). Handover decision using fuzzy MADM in heterogeneous networks, in: Proceedings of IEEE WCNC'04, Atlanta, GA, March, 2004.

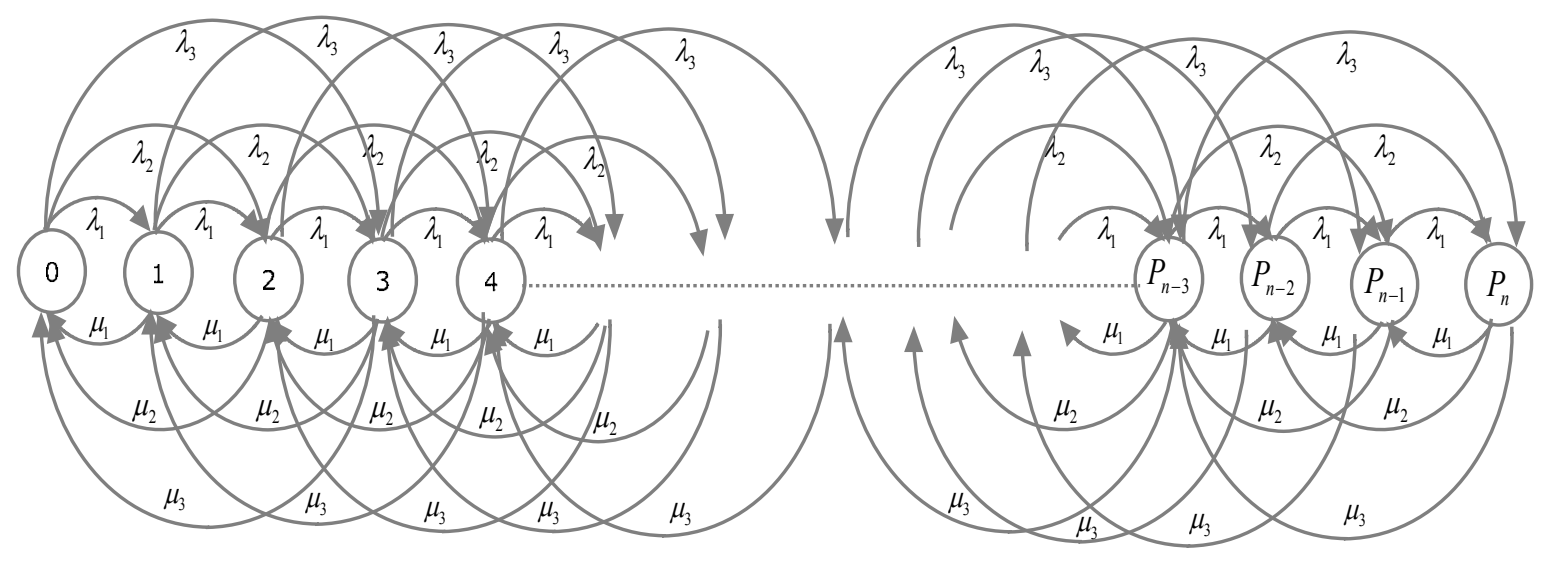

Figure 1. System Model for Call Admission Control 


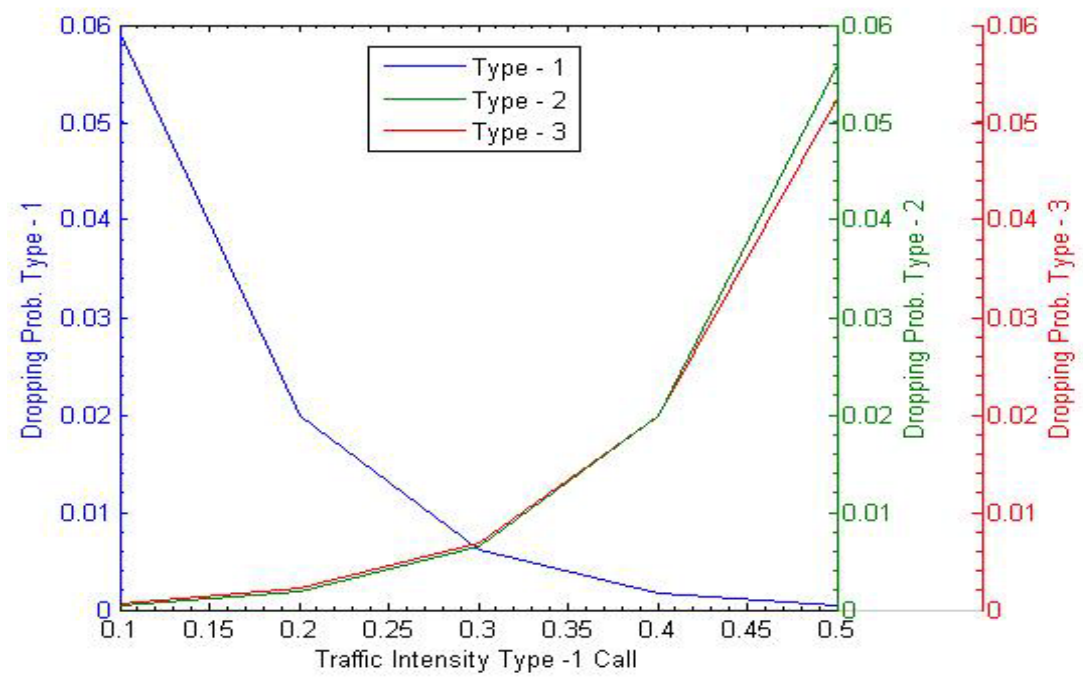

Figure 2. Call blocking probablity for varying traffic 